\title{
MIGRANTS ET RÉFUGIÉS DANS LE MONDE MYCENÉEN? LES SOURCES ÉCRITES
}

\begin{abstract}
Abstrait. - Nous réexaminons les mentions d'étrangers dans les sources écrites mycéniennes, ainsi que la collection de noms de personnes et de démonymes, afin de déterminer si ces personnes sont des réfugiés ou des migrants, ainsi que le type de migrants auquel elles appartiennent. Cependant, la position d'un migrant ou d'un réfugié implique une «altérité» claire et visible de la société dans laquelle il immigre, ainsi que des problèmes d'intégration et un faible niveau de participation à la société; les "étrangers » mentionnés dans les textes mycéniens ne semblent pas présenter ces traits. Nous constatons que, même si la majorité d'exemples attestés sont clairs, incontestés et posent peu de problèmes, la base méthodologique de la question elle-même est problématique, car elle semble reposer sur des notions de société modernes, qui ne devraient être appliquées aux sociétés prémodernes que avec une prudence extrême.
\end{abstract}

0. Les témoignages archéologiques et écrits affirment que la civilisation mycénienne - à l'instar de la civilisation minoenne qui l'a précédée - a maintenu des liens prospères et substantiels avec d'autres civilisations de la Méditerranée orientale. Ceci est certifié par des preuves écrites égyptiennes ${ }^{1}$ et hittites. ${ }^{2}$ Après avoir fondé des emporia

${ }^{1} \mathrm{Au}$ cours de la 42ème année de règne de Thoutmôsis, les scribes royales enregistrent des cadeaux pour le pharaon, y compris un vase en argent, oeuvre d' un artisan crétois; c'est par conséquent la première mention du pays lointain de Tnyw, généralement lié aux Danaioi. Cf. E. H. Cline, Sailing the Wine-Dark Sea, Oxford 1994, 110; pour une connexion possible du terme avec le hydronyme i.-e. *danu, v. M. Durante, Sulla preistoria della tradizione poetica greca, Roma 1971. Le socle d'une statue datant d'Amenhotep III contient une liste de noms de lieux dans les pays de Kftw (Crète) et Tnyw, parmi lesquels sont mentionnés, avec divers degrés de certitude, Amnisos ()mniš), Phaistos (byš[.]y), Kydonia (ktwny), Mycene (mwkinw), Thebe, Messene (midni), Nauplia (nwpiriy), Kythera (ktir), Knossos (knywš), Lyctos (rikti) et Ilios (wiriy); cf. E. Cline, 1994, 38 sqq., 112, 115.

2 v. T. Bryce, Letters of the Great Kings of the Ancient Near East. The Royal Correspondence of the Late Bronze Age, Routledge 2004, 191-203; cf. C. B. Rose, « Separating Fact from Fiction in the Aiolian Migration » Hesperia 77/3, 2008, 399-432; J. Harmatta, «Zur Ahhiyawa-Frage», in A. Bartonek (ed.), Studia Mycenaea: Proceedings of the Mycenaean Symposium (Brno, April 1966), Brno 1968, 117-124. 
à Chypre, les Grecs mycéniens ont établi des liens directs avec le cercle culturel du Proche-Orient et ont parvenu à consolider leurs contacts avec les centres commerciaux essentiels de la Méditerranée orientale, à savoir les ports phéniciens de Tyr, Ougarit et Byblos. ${ }^{3}$ De plus, les recherches linguistiques comparatives ont montré que des dizaines de mots helléniques, et de nombreux toponymes, n'ont pas d'étymologie indo-européenne évidente. Les suffixes -nth(os) et -ss(os), attestés en grec mycénien dans des mots comme asaminthos, Kuparissos, Knossos, etc., sont fréquemment discutés pour avoir une origine anatolienne, ${ }^{4}$ pour correspondre à des suffixes en langue hittite, ${ }^{5}$ ou pour avoir racines dans un substrat méditerranéen du Bronze ancien. ${ }^{6}$

1. Définir et explorer les relations entre des États et cultures voisines est, néanmoins, la partie facile de notre problème; d'autre part, définir l'émigration et l'immigration, ainsi qu'indiquer des migrants et des réfugiés dans des sociétés (supposément) étrangères, constitue, à coup sûr, la partie la plus difficile. Il y a plusieurs raisons à cela. En premier lieu, l'incapacité de relier les découvertes archéologiques aux témoignages écrits pose un problème pour définir avec précision le temps et les causes de la migration. Dans le cas des civilisations minoenne et mycénienne, malgré plusieurs tentatives vaillants, l'écriture minoenne n'a pas encore été déchiffrée, alors que toutes les essais de déchiffrement visent également à découvrir l'origine ethnique de ses créateurs; il y a, par exemple, l'hypothèse d'une origine anatolienne, particulièrement fondée sur des analyses linguistiques et des associations avec les langues hittite et louvite. ${ }^{7}$ Toutefois, comme l'a averti C. D. Buck dès 1926, elles ne reposent pas sur des preuves internes des écritures elles-mêmes, mais exclusivement sur des indications de relations culturelles entre les populations des deux côtés de la mer Égée; ${ }^{8}$

${ }^{3}$ M. L. West, 1997, 1-60.

${ }^{4}$ O. Szemerényi, «The Origins of the Greek Lexicon: Ex oriente lux ». The Journal of Hellenic Studies 94, 1974.

${ }^{5}$ Ibid.; cf. W. Burkert, 1985, 19.

${ }^{6}$ V. J. Melaart, «Bronze Age and Earlier Languages of the Near East» in Festschrift W. F. Grimm, Archaeological Theory and Practice, 1973, 163-172; cf. O. Szemerényi, 1974, n. 48.

${ }^{7}$ S. Davies (The Decipherment of the Minoan Linear A and Pictographic Scripts, Johannesburg 1967) et plusieurs autres sont en faveur de la théorie d'origine anatolienne, en tant que l'hypothèse étrusque reste influente, notamment en accord avec les hypothèses d'origine anatolienne de la langue étrusque; $c f$., in primis, O. Carruba, "L'origine degli etruschi: il problema della lingua » in Paleontologia linguistica. Atti del VI convegno internazionale di linguisti, Brescia, 1977, 137-153; G. M. Facchetti, M. Negri, Creta minoica. Sulle tracce delle più antiche scritture d'Europa, Firenze 2003; G. Barker \& T. Rasmussen, The Etruscans, London: Blackwell, 2008; et surtout R. E. Wallace, «Language, Alphabet, and Linguistic Affiliation », in S. Bell and A. A. Carpino, A companion to the Etruscans, Wiley-Blackwell, 2016.

${ }^{8}$ C. D. Buck, « The language situation in and about Greece in the second millennium $\mathrm{BC}$, » $C P h$ 21, 1926, 4-5: « La forte suspicion que la langue de ces documents crétois est liée aux langues de l'Asie mineure ne repose donc pas sur des preuves internes, tirées 
donc, ces données sont insuffisantes. Y. Duhoux souligne que nous ne pouvons pas certifier la possible origine anatolienne de l'écriture crétoise même en abordant le problème sur la base de proximité culturelle. ${ }^{9}$ Bien qu'il existe des influences discernables provenants non seulement d'Anatolie, mais aussi d'autres régions de la Méditerranée orientale, la culture minoenne possède des caractéristiques particulières et autonomes: art et artisanat originaux, architecture, système d'écriture, ainsi que manifestations religieuses qui ne peuvent en aucune manière être expliquées par des implants étrangers. ${ }^{10}$

2. La datation de l'arrivée des tribus indo-européennes dans le bassin égéen est encore plus problématique. Alors que les opinions des savants couvrent une période allant de 2300 (voire plus tôt) à $1600 \mathrm{av}$ J.-C., la date conventionnellement acceptée se situe autour de 1900 av. J.-C. La langue proto-grecque est déjà attestée au XVe siècle av. J.-C., dans les textes écrits en Linéaire B. ${ }^{11}$ Il est possible que cette langue ait été parlée à Mycènes depuis la période des tombes à fosse; pourtant, il est très problématique de postuler avec certitude qu'il s'agit du langage des habitants d'Eutrésie ou de Lerna. ${ }^{12}$ Les hypothèses principales sont construites autour des périodes de destruction et de débuts nouveaux, par exemple vers 2200 av. J.-C., après la chute de Troie II, lorsque Eutrésie et la « Maison des Tuiles » à Lerna furent confrontés au même sort. Il faut aussi garder à l'esprit la modalité des migrations d'autres peuples indo-européens, tels que les Hittites et les Louvites en Anatolie. ${ }^{13}$ Il faut certainement rester ouvert à la possibilité d'une pénétration progressive des éléments ethniques indo-européens, car il n'y a ni changements spectaculaires en ce qui concerne le caractère ethnique du monde égéen dans son ensemble, ni des traces de céramique indo-européenne, encore moins proto-grecque. Les récits de migration de masse (et donc d'immigration de masse) ne tiennent pas la route, car nous ne pouvons ni donner de date exacte à l'émergence des Hittites en Anatolie, ni expliquer de manière satisfaisante leur disparition soudaine d'un millénaire plus tard.

des documents eux-mêmes, mais sur des indices de relations culturelles entre la population crétoise primitive et celle d'Asie mineure, et sur les preuves des noms de lieux en Crète ainsi que dans d'autres parties de la mer Égée, y compris la Grèce continentale. »

9 «La langue du linéaire A est-elle anatolienne?» in M. Mazoyer, O. Casabonne (ed.), Antiquus Oriens: Mélanges offerts au Professeur R. Lebrun I, Paris 2004, 207-228.

${ }^{10}$ Ibid. 220.

${ }^{11}$ La position conventionnelle, selon laquelle la première attestation du Linéaire B devrait être datée au XIVe siècle av. J.-C., a d'abord été remise en question par de nouvelles analyses archéologiques (v. J. Gulizio, K. Pluta, T. Palaima, "Religion in the Room of the Chariot Tablets", in Potnia 2001, 453-461), et a finalement été abandonné après la découverte d'un fragment de tablette à Iklaina en Messénie en 2009; le fragment est daté au périodes HR IIB / IIIA1 / début de HR IIIA2, ce qui en fait la plus ancienne tablette stratifiée de la Grèce continentale.

$12 v$. W. Burkert 1985, 16.

13 v. L. R. Palmer 1961, 232 ss. 
À la fin du MR IB ( 1490/1470 av. J.-C.), l'île de Crète est gravement dévastée. Certains éléments indiquent que les sites avaient été abandonnés avant leur destruction et qu'ils n'avaient pas été réoccupés avant un siècle environ; les dégâts les plus graves sur les sites épuisés ont été concentrés dans les centres administratifs, ce qui atteste du caractère anthropique des destructions. Cette prétendue « occupation mycénienne » de la Crète s'inscrivait parfaitement dans notre récit, notamment parce que l'analyse prosopographique a montré que les tablettes relatives à la guerre et à la défense, découvertes dans la «Chambre aux tablettes de char », contiennent surtout des noms personnels mycéniens, ${ }^{14}$ alors qu'une analyse générale des noms de personnes attestée sur les tablettes de Crète a montré que la majorité des noms étaient non-mycéniens, ${ }^{15}$ ce qui contraste nettement avec le continent. Ainsi, en dépit de la critique de l'hypothèse d'une «occupation indoeuropéenne», laquelle est devenu particulièrement vigoureuse au cours des dernières décennies ${ }^{16}$ la hypothèse est restée très influente dans les modèles archéologiques d'interprétation de la discontinuité du Minoen Récent. Cependant, des recherches récentes ne révèlent aucune trace de population étrangère dans les tombes de MRII-IIIA de Knossos, habituellement associées aux Mycéniens, et rejettent spécifiquement la possibilité que les individus enterrés aient pu venir de l'Argolide, comme il n'y a aucune trace des gènes qui auraient dû être détectables dans la morphologie squelettique. ${ }^{17}$ Par ailleurs, l'analyse a montré une continuité dans l'histoire biologique de la population de Knossos du Bronze moyen au Bronze récent. Ces résultats, exacts de par leur nature même, ne sont pas conciliables avec la théorie d'une invasion de la Crète par les Mycéniens et de la domination politique de Knossos dans le MRIB; au contraire, ils montrent que les «sépultures de guerriers», les «sépultures avec des bronzes» ou bien les tombes à l'architecture spécifique pour le continent ne doivent pas nécessairement être associées aux Mycéniens et font possiblement partie d'un phénomène culturel plus étendu.

Avec toutes ces mises en garde à l'esprit, nous allons maintenant examiner si les documents des archives mycéniennes impliquent soit une migration, soit l'existence de migrants ou de réfugiés. ${ }^{8}$

${ }^{14} \mathrm{~J}$. Driessen, The Scribes of the Room of the Chariot Tablets at Knossos: An Interdisciplinary Approach to the Study of a Linear B Deposit, 2000; J. Gulizio, K. Pluta, T. Palaima, "Religion in the Room of the Chariot Tablets », 455 sqq.

${ }^{15}$ C. Varias García, «The Personal Names from the Knossos B-Tablets and from Mycenae Tablets », Minos 33-4, 1998-9, 349-370.

${ }^{16}$ v., e.g., Driessen and Macdonald, 1984; Preston, 2004; Nafplioti, 2008.

${ }^{17}$ Nafplioti 2012, 260.

${ }^{18}$ Nous voudrions souligner que nous n'incluons que des noms et des ethnonymes / demonymes indubitablement identifiés comme tels. Bien qu'il puisse y avoir davantage d'exemples d'étrangers dans les documents en linéaire B, nous pensons que la spéculation fondue sur des fragments aux lectures suspectes ne faciliterait pas notre analyse. 
3. Comme les tablettes mycéniennes sont en réalité des documents administratifs des archives des palais de Knossos, Pylos, Mycènes et Thèbes, les scribes ont souvent enregistré des noms de personnes, ainsi que des demonymes indiquant le lieu exact ou le domain d'origine de quiconque a été mentionné dans le texte. Un bon exemple serait la tablette KN E 777, enregistrant un groupe de travailleuses qui reçoivent des rations de blé; ces femmes étaient ko-no-si-ja [Knōssiai], a-mi-ni-si-ja [Amnisiai] and pa-i-ti-ja [Phaistiai], c'est-à-dire originaires des centres crétois bien connus de Knossos, Amnisos et Phaistos. $^{19}$

a. Nous avons trois témoignages incontestables d'étrangers provenant de l'Afrique: $a_{3}$-ku-pi-ti-jo, a $a_{3}$-ti-jo-qo and mi-sa-ra-jo.

a $_{3}$-ku-pi-ti-jo, ${ }^{20}$

$\mathrm{KN}$ Db $1105+1446$

.A

OVIS $^{\mathrm{m}} 52$

OVIS $^{\mathrm{f}} 28$

.B a3-ku-pi-ti-jo / su-ri-mo

« Aigyptios: 52 béliers, 28 brebis; percepteur, Su-ri-mo. » Le nom du berger fait une claire allusion à son origine.

$\mathbf{a}_{3}$-ti-jo-qo, ${ }^{21}$

PY Eb 846

.1 ai-ti-jo-qo e-ke-qe o-na-to ke-ke-me-na ko-to-no

.B pa-ro da-mo ko-to-no-o-ko / to-so-de pe-mo WHEAT I[T]

"Aithiok ${ }^{\mathrm{w}} \mathrm{s}$, et il détient le bail d'une parcelle commune du village (étant lui-même) propriétaire de parcelle; tellement de semences: 174 1. du blé."

\section{mi-sa-ra-jo, ${ }^{22}$}

KN F $841+867$

.4 pa-i-to mi-sa-ra-jo sa-i-ti-ni-we-jo

$.5 s u$-za FIGS 75 ka-po e-[ra-wa?

$.6 \quad e-r a]$-wa OLIVES 46 e-ra-wa

19 cf. J. Weilhartner, "The Interrelationship between Mycenaeans and Foreigners", in M. Oller, J. Pàmias, C. Varias (ed.), Tierra, territorio y población en la Grecia antigua: aspectos institucionales y míticos, Mering 2017, pp. 151-168.

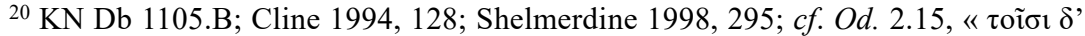

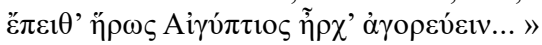

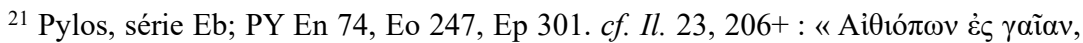

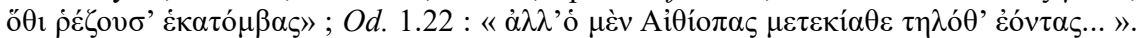
Le problème est que nous n'avons tout simplement aucune idée de ce que "Aithiops » voulait dire à l'âge du bronze. Nul doute qu'au-delà d'un certain point de l'histoire, les Grecs ont interprété le mot comme "visage brûlé» $\left(\alpha i ̂ \omega^{+*} \omega ̋ \psi\right)$. Il est fréquemment affirmé que c'est la véritable étymologie du mot, mais l'incertitude est plus grande que ne le laissent les érudits : cf. R. Beekes, "Aithiopes." Glotta 73, 1995/6, pp. 12-34; W. Kullmann, "Ilias und Aithiopis." Hermes 133, 2005, pp. 9-28.

${ }^{22}$ KN F 841.4; Cline 1994, 128. 
Cette tablette endommagée appartient à un groupe de tablettes qui laissent l'impression d'être des listes d'offrandes, mais qui pourraient bien être des listes de rations ordinaires. Les offrandes comprennent principalement du blé, des olives et des figues, dont les quantités indiquées sont considérables: 90001 . de figues et 5500 d'olives. Le nom personnel *Misraios pourrait être lié à Misr, «Égypte», bien que ce soit très difficile à déterminer, car c'est un hapax connu uniquement de Knossos.

b. Quatre autres demonymes ou ethnonymes probables font référence à des personnes originaires de la côte du Levant et de l'île de Chypre:

tu-ri-jo, ${ }^{23}$ Turios, un démonyme de la ville portuaire phénicienne de Tyr, en grec Túpıos; mentionné dans PY Jn 693, dans une liste de forgerons, ainsi que dans KN Nc 4473, avec une fonction obscure, en tant que nom personnel.

À Knossos, une po-ni-ke-ja est mentionné dans KN Ln 1568, peut-être un nom personnel féminin dans le cas datif [Phoinikeiāi?]; la tablette enregistre qu'elle a reçu de la laine, alors possiblement une tisserande. Il y a aussi un po-ni-ki-jo, ${ }^{24}$ qui peut bien être un ethnonyme.

Il y a aussi des mentions du nom personnel ki-nu-ra, ${ }^{25}$ Kinurās, éventuellement un nom sémitique. En outre, c'est le nom du légendaire roi chypriote Cinyras, évoqué dans la tradition homérique en tant que prêtre d'Aphrodite à Paphos, musicien qui a osé défier le dieu Apollon lors d'un concours musical et ancêtre mythique des Cinyrades, qui a joué de son instrument dans des temples honorant le culte paphien. Le nom lui-même est un terme sémitique pour les joueurs professionnels de cithare (kinnor). ${ }^{26}$

Le nom ku-pi-ri-jo ${ }^{27}$ Kuprios, "Chypriote", est assez ordinaire à Pylos; dans PY Cn 131, 719, il s'agit du nom d'un berger, dans Jn 320 d'un forgeron, dans l'Un 443 - d'un commerçant, ou éventuellement d'un fabricant d'onguent. Ce nom est également fréquent à Knossos, par exemple dans Fh 347+, Ga 517+, désignant possiblement un percepteur ou un fabricant d'onguent. ${ }^{28}$

${ }^{23}$ KN Nc 4473; PY Jn 693.8; Cline 1994, 129.

${ }^{24} \mathrm{KN}$ Bg 834, 992, 1021, etc.; v. Aura-Jorro, Diccionario, 139-140; cf. Yasur-Landau, 2010, 38 sqq.

${ }^{25}$ Qa $1301 ;$ Vn 865.

${ }^{26}$ Certains érudits proposent une connexion avec la divinité ougaritique mineure Kinaru, le dieu de la lyre; $c f$. J. P. Brown (1965). "Kothar, Kinyras, and Kythereia". Journal of Semitic Studies 10, pp. 197-219.

${ }^{27}$ PY Cn 719.7, Jn 320.3, Un 443.1; KN Fh347.1, Gg 995; cf. Palaima 1991, 280281; Cline 1994, 130; Shelmerdine 1998, 295.

${ }^{28}$ Dans cette section, on pourrait éventuellement inclure aussi a-ra-si-jo (un homme d'Alashiya/Alassa? KN Df 1229.B, Fh 369, X 1463; Cline 1994, 130); pe-ri-ta (un homme de Berytos? KN V 60.5; Cline 1994, 129); ainsi que a-ra-da-jo (un homme d'Arwad? KN 1516.3; Cline 1994, 124). 
c. Enfin, nous avons plusieurs démonymes d'Asie Mineure - la désignation principale étant, bien sûr, celle d' "Asiatique».

Le nom personnel a-si-wi-jo, Aswios, 'Asian', est souvent attesté, par exemple, en tant que berger dans les KN Df 1469 et PY Cn 285; dans PY Eq 146 en tant que détenteur de terre, dans MY Au 653 en tant que travailleur. Dans la variante $\mathbf{a - * 6 4 - j o , ~ e n ~ t a n t ~ q u e ~ d e s t i n a - ~}$ taire d'orge dans PY Fn 324, un messager dans PY Cn 12887, un forgeron dans PY Jn 832. À Knossos, dans Sc 261 en tant que cocher. En tant que prénom féminin, a-*64-ja est attesté par exemple, à Pylos, dans Vn $341191 .^{29}$

La présence d'une population d'Asie Mineure est encore confirmée par les nombreux témoignages de la déesse la plus mentionnée, Potnia; à Pylos (Fr 1206), elle est attestée sous la forme de po-ti-ni-ja a-si-wi-ja, "Maîtresse de l'Asie», ce qui peut impliquer une forme de syncrétisme des cultes de la mer Égée et de l'Asie Mineure.

Qu'il s'agisse de migrants, d'amis ou de captifs, nous voyons des travailleurs comme des mi-ra-ti-jo / mi-ra-ti-ja, «un homme / une femme de Milet»; ze-pu 2 -ra $\mathbf{a}_{3}$, «une femme de Halicarnassus»; ki-nidi-ja, «une femme de Cnide»; ki-si-wi-jo / ki-si-wi-ja, «un homme / une femme de Chios»; ra-mi-ni-jo / ra-mi-ni-ja, «un homme / une femme de Lemnos»; et enfin, i-ja-wo-ne, une désignation possible pour les Ioniens. ${ }^{30}$

d. Nous terminons avec une attestation de personnes originaires des îles de la mer Ionienne: za-ku-si-jo / za-ku-si-ja, «homme / femme de Zakynthos». ${ }^{31}$

4. Les maigres informations fournies par les tablettes n'offrent aucune aide pour déterminer non seulement le type de migrants auquel nous sommes confrontés - qu'il s'agisse de migrants d'élite, de roturiers ou de réfugiés - mais même si les personnes nommées sont du tout des migrants ou des réfugiés. Ils semblent être principalement des travailleurs, des forgerons et des fabricants d'onguent, même des soldats,

${ }^{29}$ a-si-wi-jo: KN Df 1469.B; PY Cn 285.12, Eq 146.11; MY Au 653.5, 657.11; Cline 1994, 130; Shelmerdine 1998, 295. a-*64-jo: KN Sc 261; PY Cn 1287.1, Fn 324.3. a-si-wi-ja: PY Fr 1206. a-*64-ja: PY Vn 1191.2, Az 701, Ab 515.B, Ad 315, 326. Cf. Aura-Jorro, Diccionario, s.v.

${ }^{30}$ mi-ra-ti-jo: TH Fq 177, 198, 214, 244, 254 + 255, 269, 276; Shelmerdine 1998, 295; Sacconi 2001, 393. mi-ra-ti-ja: PY Aa 798, 1180, Ab 382.B, 573.B, Ad 380, 689; Palaima 1991, 279-280; Cline 1994, 130. ze-pu2-ra3: PY Aa 61, Ad 664; Cline 1994, 130. ki-ni-di-ja: PY Aa 792, Ab 189.B, An 292.4, Ad 683; Cline 1994, 130. ki-si-wi-jo: KN V 60.2. ki-si-wi-ja: PY Aa 770, Ab 194.B, Ad 675. ra-mi-ni-jo, ra-mi-ni-ja: PY Ab 186.B, An 209.2; i-ja-wo-ne: KN B 164.4, Xd 146.6; Aura-Jorro, Diccionario 1985: 237.

${ }^{31}$ za-ku-si-jo / za-ku-si-ja: PY An 610, Sa 751, 787.B, MY Oe 122; Palaima 1991, 281. On pourrait y ajouter ko-ro-ku-ra-i-jo, 'un homme de Corcyre?', PY An 641.4, 656.7, 756.18, 661.4-6, Na 396, 405, 510; mais cf. Yasur-Landau, 2010. 
mais aussi des propriétaires terriens, ce qui implique une position pleinement différente dans la société mycénienne. ${ }^{32}$

On est tenté de supposer que ce qu'on voit sur les tablettes sont des migrants économiques, car leurs démonymes sont mentionnés sur des tablettes indiquant les activités économiques du palais. Cependant, cela ne veut pas dire grand-chose, car il s'agit du seul type de tablettes à notre disposition. D'autre part, une partie de ces migrants semble jouir d'un statut élevé dans la société mycénienne, ce qui implique qu'il pourrait s'agir de réfugiés d'élite, appartenant aux couches sociales les plus élevées de leur pays. De plus, les documents écrits ne révèlent pas les causes des migrations prétendues. C'est précisément pour cette raison qu'il existe une littérature volumineuse sur le sujet indiqué dans le titre. Une partie en est fondée uniquement sur avis personnel; une autre, sur des faits scientifiques; mais la plus grande partie est simplement une spéculation déductive appuyée sur une couche étroite de données factuelles. Les textes peuvent vouloir dire quelque chose - ou bien rien - que en fonction de l'interprétation des données collatérales.

L'état et la société mycéniennes étaient établis sur le pouvoir, manifesté par les palais et les villas palatiales, symboles de l'autorité hautement visibles et stratégiquement placés; une autre manifestation du pouvoir était la capacité d'enrôler des ressources pour la construction de navires, la redistribution des revenus, l'approvisionnement en fêtes et festivals religieux et la conduite des échanges. Bien que nous n'ayons aucune affirmation concrète et tangible d'une flotte de guerre, nous disposons de nombreuses rapports d'importation et de mobilité d'artistes et de diplomates, qui ont dû voyager par mer. ${ }^{33}$

C'est là que l'idée de la mer en tant qu'agent de liaison entre dans l'expérience historique. Comme le suggère Hodos, ${ }^{34}$ nous devrions aborder le modèle de mondialisation en démarrant non d' une idée d'agent de compression, dans laquelle le monde est compris comme un lieu cohérent et culturellement unifié, mais plutôt comme un instrument permettant de relier les hétérogénéités culturelles à un système logique, qui fonctionne tout de même correctement. Dans ce système, les pratiques partagées ne sont pas des pratiques identiques; 1'intensité du contact n'implique pas nécessairement des gagnants et des perdants, mais peut conduire à des tentatives de redéfinir les frontières entre les groupes sociaux. En d'autres termes, les changements qui surviennent après des contacts prolongés avec des cultures compatibles peuvent

${ }^{32}$ Des fragments de fresques à la « Maison des Fresques » du palais de Knossos représentent des soldats nubiens en train de faire exercice (?) avec des Mycéniens; $c f$., cependant, S. Hood, "Dating the Knossos frescoes », in Aegean Wall Painting: a Tribute to Mark Cameron, British School at Athens Studies v. 13, 2005, pp. 45-81.

${ }^{33}$ Hitchcock \& Maeir 2018, 3; cf. Wachsmann 2008, 128.

${ }^{34}$ Hodos, T. (2010). "Globalization and Colonization: A View from Iron Age Sicily." Journal of Mediterranean Archaeology 23.1, pp. 81-106. 
tout au plus être des réactions qui cherchent à redécouvrir les particularités, le localisme et la différenciation, mais non la tendance aux conflits.

Dans un tel système, les groupes ethniques et les collectivités ne sont des catégories civiques ni innées, ni essentielles. Comme nous l'avons déjà vu plus à gauche, pratiquement aucun groupe ethnique de l'époque mycénienne ne pourrait être identifié par association exclusive avec un certain type de poterie, un vêtement particulier, une forme particulière de rituel funéraire ou un dialecte spécifique. ${ }^{35}$ L'identité ethnique n'est pas un fait naturel de la vie, mais un élément qui doit être activement proclamé par des canaux discursifs. Ce qui la distingue des autres identités sociales est sa dimension historique, à savoir la conscience qui fournit la réponse à des questions telles que «qui sommes-nous», «comment sommes-nous arrivés ici» et enfin «qu'estce qui nous différencie des autres». ${ }^{36}$ Mais le phénomène de l'ethnicité, qui a suscité un intérêt considérable de la part des archéologues, reste complexe et fait l'objet de nombreux débats. Les désignations ethniques des sociétés historiques ont souvent été attribuées sans discernement et sans accorder une attention systématique aux processus ethniques. Il a été supposé que les groupes ethniques pourraient être définis objectivement par des aspects de spécificité culturelle, linguistique et raciale; mais dans des cas aussi lointains et peu attestés que celui de la civilisation mycénienne, on ne peut jamais savoir avec certitude si un aspect particulier attesté in situ définit l'appartenance ethnique ou culturelle. Les limites sont très rarement tranchées. Par exemple, Patterson définit un groupe culturel comme un groupe de personnes partageant un ensemble identifiable de significations, de symboles, de valeurs et de normes, tandis qu'un groupe ethnique conserve de plus une conscience de son appartenance et une identité de groupe consciente. Un groupe culturel, ou des segments de celui-ci, peuvent devenir des groupes ethniques, mais uniquement lorsqu'ils remplissent les conditions d'ethnicité, quelle que soit leur forme. ${ }^{37}$

5. Ceci est très important, car pour représenter les migrants ou les refugiés tels que nous les définissons de nos jours - et, par conséquent, pour être clairement visibles dans nos sources - les personnes déplacées doivent remplir une condition très claire: celle d'une « altérité » visible. Mais les conceptions de l'ethnicité à l'âge du bronze

${ }^{35}$ Hall, J. M. (1997). Ethnic Identity in Greek Antiquity. Cambridge University Press, 182 sqq.

${ }^{36}$ Incidemment, cela peut être la raison même de l'existence des mythes d'origine ethnique, qui servent à proclamer l'identité ethnique d'un groupe; comme Hall (1997) l'a fait remarquer avec lucidité, le fait que de tels mythes existent dans un certain nombre de variantes parfois contradictoires n'est pas la conséquence d'un souvenir d'un passé préhistorique qui a commencé à se dégrader, mais une indication de l'interaction dynamique et complexe entre les positions et des contre-positions ethniques, qui se produisent de manière intermittente tout au long de la période historique.

${ }^{37}$ Patterson 1975, 309-10; cf. Feuer 2011, 508 sqq. 
égéen différaient à peu près sûrement de celles d'aujourd'hui. L'ethnicité a certainement eu des ramifications politiques différentes en l'absence de l'État-nation moderne, et il est probable qu'il existait également d'autres différences moins perceptibles. Davis et Bennet vont jusqu'à affirmer que « dans une large mesure, qui pouvait ou ne pouvait pas devenir mycénien devait dépendre de la mesure dans laquelle un groupe particulier était incorporé dans le système politique et économique, dominé par l'élite du palais mycénien »; auparavant, Killen avait affirmé que les «percepteurs » des tablettes pouvaient constituer une élite panégéenne mobile, utilisant une liste partagée de noms d'élites, peut-être avec des liens de parenté entre des élites situées à des endroits différents. ${ }^{38}$

Pour faire court: compte tenu de tout ce qui précède, bien qu'il soit indiqué par un demonyme dans les textes, une personne originaire de Milet, Halicarnasse ou Cnide ne pouvait guère être vue comme un étranger ou un migrant d'outre-frontière culturelle; à en juger par la facilité relative avec laquelle ils étaient devenus partie intégrante du système de production palatial, on pourrait en dire autant des Chypriotes, des Zakynthiens, même des Tyriens ou des Sidoniens. Il n'est même pas nécessaire de commenter le cas des percepteurs d'élite et des propriétaires terriens.

6. Lorsque les palais mycéniens ont finalement été abandonnés, le contrôle mycénien sur les routes commerciales s'est effondré; à moins d'être bien défendus, les littoraux, les ports naturels et les vallées fluviales s'ont trouvés susceptibles d'attaques. Les habitants des zones vulnérables aux attaques et à la piraterie étaient motivés à se déplacer vers des lieux moins accessibles et plus faciles à défendre, situés plus à l'intérieur des terres.

Cet abandon et cette migration vers des zones moins accessibles sont la caractéristique principale de nombreux établissements, clairement visibles dans les données archéologiques. ${ }^{39}$ Peu importe si nous sommes disposés à attribuer ces attaques à des assaillants énigmatiques, connus sous le nom de «peuples de la mer», ${ }^{40}$ ou à les examiner

38 J. L. Davis \& J. Bennet 1999, 113; Killen, 1979. Cf. Feuer, 2011.

${ }^{39}$ Parmi des dizaines de volumes de littérature savante, nous ne référons qu'aux oeuvres générales les plus récentes ou influentes: R. Drews, The End of the Bronze Age: changes in warfare and the catastrophe ca. 1200 B.C., Princeton University Press, 1993; O. Dickinson, The Aegean from Bronze Age to Iron Age: Continuity and Change between the Twelfth and Eighth Centuries BC, London-New York, 2006; G. D. Middleton, The Collapse of Palatial Society in LBA Greece ad the Postpalatial Period, Oxford 2010; E. H. Cline, 1177 B.C.: The Year Civilization Collapsed, Princeton University Press, 2014.

${ }^{40}$ L'expression «peuples de la mer » trouve son origine dans un texte sur le second pylône du temple de Médinet Habou, célèbrant la victoire de Ramsès III dans la guerre contre les «peuples de la mer ", qui mènent deux vagues d'assauts contre le Nord de l'Égypte; $v$. W. F. Edgerton, J. Wilson, Historical Records of Ramses III: The Texts in Medinet Habu, vol. I-II, translated with explanatory notes, Chicago 1936. 
en tant que phénomènes distincts et locaux; ce qui s'est passé à cette époque a clairement inspiré des migrations et a accéléré le processus d'établissement de nouvelles réalités et pratiques sociales: ${ }^{41}$ perte de complexité sociale, disparition de nombreuses pratiques artisanales ${ }^{42}$ transformations dans l'environnement culturel, et surtout une notion excessivement soulignée de «nous contre les autres», facilitant et dépêchant l'évolution sociétale d'une société fondée sur des conformités culturelles, à une société créée sur des prémisses ethniques.

En fin de compte, cela conduira la société humaine au concept d'État-nation; et c'est précisément l'influence du modèle conceptuel idéalisé de l'État-nation - lequel a commencé à prendre forme à l'époque de la Révolution française et a été élaborée en pratique politique et réflexion théorique au cours du XIXe siècle - que nous semblons incapables de nous débarrasser en essayant d'analyser les sociétés prémodernes, y compris la mycénienne. Pourtant, si nous parvenons à voir le tableau dans son ensemble, de manière totalement indépendante des notions de société modernes, on peut se demander s'il serait approprié de rechercher des migrants dans les sources écrites mycéniennes.

\section{BIBLIOGRAPHIE SÉLECTIVE}

Barker, G. \& Rasmussen T. (2008). The Etruscans. London: Blackwell.

Bennet, J. (1999). "The Meaning of 'Mycenaean': Speculations on Ethnicity in the Aegean Late Bronze Age." Mycenaean Seminar Abstract. Bulletin of the Institute of Classical Studies 43, 224.

Bryce, T. (2004). Letters of the Great Kings of the Ancient Near East: The Royal Correspondence of the Late Bronze Age. London: Routledge.

Buck, C. D. (1926). "The language situation in and about Greece in the second millennium BC," CPh 21, 1-26.

Chapman, R (2005). "Changing Social Relations in the Mediterranean Copper and Bronze Ages". In E. Blake \& A. B. Knapp (eds.). The Archaeology of Mediterranean Prehistory. Oxford and Malden: Blackwell, pp. 77-101.

Cline, E. H. (1994). Sailing the Wine-dark Sea: International Trade and the Late Bronze Age Aegean. BAR International Series 591. Oxford: Tempvs reparatvm.

Cline, Eric H. (2014). 1177 B.C.: The Year Civilization Collapsed. Princeton University Press.

${ }^{41} v$. Crielaard 2011, 96.

42 Il semble que la gloire passée n'a été vaguement rappelée que par les aoidoi des âges obscurs; le reste a été oublié jusqu'à ce qu'il soit mis au jour par les archéologues modernes. V. Claude Mossé, La Grèce archaïque d'Homère à Eschyle, Seuil, 1990 ; JeanClaude Poursat, La Grèce préclassique, Des origines à la fin du vie siècle, Seuil, 1998. 
Coleman, J. (2000). "An Archaeological Scenario for the 'Coming of the Greeks' ca. 3200 B.C." The Journal of Indo-European Studies 28, pp. 101-154.

Crielaard, J.-P. (2011). "The 'Wanax to Basileus Model' Reconsidered: Authority and Ideology After the Collapse of the Mycenaean Palaces". In A. Mazarakis Ainian (ed.), The "Dark Ages" Revisited: Acts of an International Symposium in Memory of William D.E. Coulson, University of Thessaly, Volos, 14-17 June 2007. Volos: University of Thessaly Press, pp. 83-111.

Davis, S. (1967). The Decipherment of the Minoan Linear A and Pictographic Scripts. Johannesburg: Witwatersrand University Press.

Davis, J. L. \& Bennet, J. (1999). "Making Mycenaeans: Warfare, Territorial Expansion, and Representations of the Other in the Pylian Kingdom." In R. Laffineur (ed.), Polemos: Le contexte guerrier en Égée à l'âge du Bronze. Actes de la 7e Rencontre égéenne internationale. Aegaeum 19. Liège and Austin.

Dickinson, O. (2006). The Aegean from Bronze Age to Iron Age: Continuity and Change between the Twelfth and Eighth Centuries BC. London-New York.

Drews, R. (1993). The End of the Bronze Age: changes in warfare and the catastrophe ca. 1200 B.C. Princeton University Press, 1993.

Drews, R. (2017). Militarism and the Indo-Europeanizing of Europe. London: Routledge.

Driessen, J., Macdonald, C.F. (1984). "Some military aspects of the Aegean in the late fifteenth and the early fourteenth centuries B.C." Annual of the British School at Athens 79, pp. 49-70.

Driessen, J. (2000). The scribes of the room of the chariot tablets at Knossos: Interdisciplinary approach to the study of a Linear B deposit. Salamanca: Ediciones Universidad de Salamanca.

Дуев, Р. (2009). Музиката во микенскиот свет и Хомер. Скопје: Силсонс.

Duhoux, Y. (2004). «La langue du linéaire A est-elle anatolienne? » in M. Mazoyer, O. Casabonne (ed.), Antiquus Oriens: Mélanges offerts au Professeur René Lebrun I, Paris 2004, 207-228.

Feuer, B. (2011). "Being Mycenaean: A View from the Periphery." American Journal of Archaeology 115.4, pp. 507-536.

Gulizio, J., Pluta, K. \& Palaima, T (2001). "Religion in the Room of the Chariot Tablets." In R. Hägg and R. Laffineur (eds)., Potnia (Aegaeum 22). Liège and Austin, pp. 453-461.

Hall, J. M. (1997). Ethnic Identity in Greek Antiquity. Cambridge University Press.

Hitchcock, L. A. \& Maeir, A. (2018). "Pirates of the Crete-Aegean: Migration, Mobility, and Post-Palatial Realities at the End of the Bronze Age". Proceedings of the 12th International Conference of Cretan Studies, Heraklion, 21-25 September 2016. Heraklion: Cretan Historical Society, pp. 1-12.

Hodos, T. (2010). "Globalization and Colonization: A View from Iron Age Sicily." Journal of Mediterranean Archaeology 23.1, pp. 81-106.

Meiri, M. et al. (2019). "Mobility and trade in Mediterranean antiquity: Evidence for an 'Italian connection' in Mycenaean Greece revealed by ancient DNA of livestock." Journal of Archaeological Science: Reports 23, pp. 98-103.

Melaart J. (1973), "Bronze Age and Earlier Languages of the Near East." Festschrift W. F. Grimm, Archaeological Theory and Practice, 1973, 163-172.

Middleton, G. D. (2010). The Collapse of Palatial Society in LBA Greece ad the Postpalatial Period. Oxford: Clarendon Press.

Mossé, C. (1990). La Grèce archaïque d'Homère à Eschyle. Seuil, coll. « Points Histoire $»$.

Nafplioti, A. (2008). "Mycenaean political domination of Knossos following the LMIB destructions on Crete: negative evidence from strontium isotope ratio analysis $\left({ }^{87} \mathrm{Sr} /{ }^{86} \mathrm{Sr}\right)$ ". Journal of Archaeological Science 35, pp. 2307-2317.

Nafplioti, A. (2012). "Late Minoan IB destructions and cultural upheaval on Crete: A bioarchaeological perspective". In E. Kaiser, J. Burger and W. Schier (eds.). Population 
Dynamics in Prehistory and Early History. New Approaches by Using Stable Isotopes and Genetics. Berlin-Boston: De Gruyter, pp. 241-263.

Patterson, O. (1975). "Context and Choice in Ethnic Allegiance: A Theoretical Framework and Caribbean Case Study." In N. Glazer and D.P. Moynihan (eds.), Ethnicity: Theory and Experience, Cambridge: Harvard University Press, pp. 305-349.

Poursat, J.-C. (1998). La Grèce préclassique, Des origines à la fin du vie siècle. Seuil, coll. « Points Histoire ».

Preston, L. (2004). "A mortuary perspective on political changes in Late Minoan II-IIIB Crete." American Journal of Archeology 108, pp. 321-348.

Pullen, D. (2008). "The Early Bronze Age in Greece". In C. W. Shelmerdine (ed.), The Cambridge Companion to the Aegean Bronze Age. Cambridge University Press.

Rose, C. B. (2008). "Separating Fact from Fiction in the Aiolian Migration." Hesperia 77.3, pp. 399-432.

Shelmerdine, C. W. (1998). "Where Do We Go from Here? And How Can the Linear B Tablets Help Us Get There?" In E. Cline \& D. Harris-Cline (eds.). The Aegean and the Orient in the Second Millennium (Aegaeum 18). Liège: 292-298.

Tandy, D. W. (1997). Warriors into Traders. The Power of the Market in Early Greece. University of California Press.

Van De Mieroop, M. (2009). The Eastern Mediterranean in the Age of Ramesses II. Wiley-Blackwell.

Varias García, C. (1999). "The Personal Names from the Knossos B-Tablets and from Mycenae Tablets." Minos 33-4, , pp. 349-370.

Wachsmann, S. (2008). Seagoing Ships and Seamanship in the Bronze Age Levant. Ed Rachal Foundation Nautical Archaeology Series. Texas A\&M University Press.

Wallace, R. E. (2016). "Language, Alphabet, and Linguistic Affiliation”. In S. Bell and A. A. Carpino, A companion to the Etruscans. Wiley-Blackwell.

Weilhartner, J. (2017). "The Interrelationship between Mycenaeans and Foreigners". In M. Oller, J. Pàmias, C. Varias (eds.), Tierra, territorio y población en la Grecia antigua: aspectos institucionales y míticos, Mering: Utopica Verlag, pp. 151-168.

West, M. L. (1997). The East Face of Helicon: West Asiatic Elements in Greek Poetry and Myth. Oxford: Clarendon Press. 
